\title{
How Users Search the Mobile Web: A Model for Understanding the Impact of Motivation and Context on Search Behaviors
}

Citation: Dan Wu, Man Zhu \& Aihua Ran (2016). How Users Search the Mobile Web: A Model for Understanding the Impact of Motivation and Context on Search Behaviors. Received: Jan. 3, 2016 Revised: Mar. 2, 2016 Accepted: Mar. 3, 2016

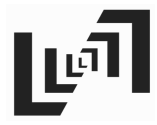

JDIS

Journal of Data and Information Science Vol. 1 No. 1, 2016 pp 98-122 DOI: $10.20309 /$ jdis. 201608

\author{
Dan $\mathrm{Wu}^{\dagger}$, Man Zhu \& Aihua Ran
}

School of Information Management, Wuhan University, Wuhan 430072, China

\section{Abstract}

Purpose: This study explores how search motivation and context influence mobile Web search behaviors.

Design/methodology/approach: We studied 30 experienced mobile Web users via questionnaires, semi-structured interviews, and an online diary tool that participants used to record their daily search activities. SQLite Developer was used to extract data from the users' phone logs for correlation analysis in Statistical Product and Service Solutions (SPSS).

Findings: One quarter of mobile search sessions were driven by two or more search motivations. It was especially difficult to distinguish curiosity from time killing in particular user reporting. Multi-dimensional contexts and motivations influenced mobile search behaviors, and among the context dimensions, gender, place, activities they engaged in while searching, task importance, portal, and interpersonal relations (whether accompanied or alone when searching) correlated with each other.

Research limitations: The sample was comprised entirely of college students, so our findings may not generalize to other populations. More participants and longer experimental duration will improve the accuracy and objectivity of the research.

Practical implications: Motivation analysis and search context recognition can help mobile service providers design applications and services for particular mobile contexts and usages.

Originality/value: Most current research focuses on specific contexts, such as studies on place, or other contextual influences on mobile search, and lacks a systematic analysis of mobile search context. Based on analysis of the impact of mobile search motivations and search context on search behaviors, we built a multi-dimensional model of mobile search behaviors.

Keywords Mobile search; Query formulation; Search motivation; Search context; Contextual dimension

Corresponding author: Dan Wu (E-mail: danwoo@whu.edu.cn). 


\section{Introduction}

With the development of the mobile Internet, mobile search has become an important means for people to access information. According to the $37^{\text {th }}$ Statistical Report on Internet Development in China ${ }^{\circledR}$, by the end of December 2015, the number of Chinese mobile users had reached 620 million, $90.1 \%$ of total Internet users. Furthermore, mobile search applications are used by $77.1 \%$ of mobile users in 2015 , at an annual growth rate of $11.3 \%$, up 48.7 million from 429 million in 2014. In the usage of mobile applications, mobile search ranks third after instant communication and news applications. College students are the major users of mobile search.

As the popularity of mobile search among young people continues to grow, many mobile service providers have engaged with mobile search applications development to attract college students. In order to design applications and services for particular information needs and mobile contexts, it is necessary to understand how user motivation and context influence their search behaviors. To this end, we conducted an online diary study with college students. Our research questions are:

RQ 1: How do mobile search motivations influence mobile search behaviors? Do mobile search sessions driven by different motivations relate to each other?

RQ 2: How does mobile search context influence mobile search behaviors? What is the relationship between different context dimensions?

Based on the investigation results we developed a mobile search behavior model to understand the relations between different variables.

\section{Related Work}

\subsection{Research on Mobile Search Motivations}

Malhotra, Galletta, and Kirsch (2008) formed a theoretical basis for discerning whether user behaviors resulted from perceived external influences or personal volition. Since then, user motivation has been investigated as an important influencing factor in many information-seeking behavior studies. Chang and Yang (2009) applied motivation theory to participating behaviors, Bilandzic and Foth (2013) to social learning behavior, and Kim and Adler (2015) to online information sharing behavior. However, these studies did not take into account the device where mobile search behaviors happened, omitting comparisons between mobile and desktop search.

Recent studies investigate how user motivation affects information behavior in the mobile context. Ciampa (2014) examined whether and how Malone and Lepper's

\footnotetext{
(1) http://www.cnnic.cn/hlwfzyj/hlwxzbg/201601/P020160122469130059846.pdf
}

Journal of Data and Information Science http://www.jdis.org 


\section{Research Paper}

taxonomy of intrinsic motivations for learning applied equally well to mobile learning, and Huizenga et al. (2009) applied motivation theory to mobile gamebased learning. Church and Oliver (2011) studied many aspects of mobile search, and proposed a motivation classification theory to help understand why users use mobile devices to search for information. They treated search motivations as a part of mobile search behaviors, rather than a driving factor of mobile search activity, and indicated that more effort is needed to study the relationship between mobile search and information-seeking motivation.

\subsection{Research on the Mobile Search Context}

Search context was identified as one factor that plays a vital role in mobile search behavior. Kong et al. (2015) proposed two types of short-term context: pre-search context and in-search context, and suggested that pre-search context triggers the search. Liu and $\mathrm{Wu}$ (2015) suggested that contextual information, which included time, weather, and emotions, influences a user's preference on a certain point of interest. Taking geographical place and user behavior as contextual information, Kiseleva (2015) studied their influence on searching and browsing behavior. Gasimov, Magagna, and Sutanto (2010) divided contextual information into device information, user information, and environment information, and presented a simple architecture for adaptive mobile Web page browsing.

Recent studies discuss the effect of mobile devices on mobile search behavior. Church and Oliver (2011) suggested that limitations in screen size and the type of interactions and input that mobile devices can support play an important role in shaping the way that mobile users use mobile search engines. Kamvar and Baluja (2006) found out that Google's PDA logs related to searches originating on PDAlike devices with sophisticated input capabilities. Park and Ohm (2014) introduced an integrated research model to examine user acceptance of mobile map services in mobile devices. Studies of mobile devices mostly focus on screen size and how it works with search behavior, such as map search behavior. Device is also considered one of multiple contextual dimensions.

Most current research focuses on specific context, however, such as studies on place, or other contextual influences on mobile search, and lacks a systematic analysis of mobile search context. In this paper, we analyze college students' mobile search behaviors in a multiple-dimensional context, and build a model of mobile search behaviors based on context.

\section{Methodology}

Journal of Data and Information Science

We conducted an online diary study, in which 30 participants agreed to share daily mobile search records on their phone logs with our researchers. The experiment 
lasted 15 days, and the participants were asked to do mobile search as usual in their daily life. An application usage-monitoring tool, based on SQLite Developer, was installed on participants' phones to record their mobile search behavior details.

\subsection{Participants}

Online questionnaires were distributed to a wide range of college students to explain our experiment and investigate the frequency of mobile search in their everyday life, the number and type of mobile applications installed in their phones, and their level of mobile search ability. We used these questionnaires to analyze the background information of possible participants. We decided to select skilled users to participate in our experiment for two reasons. One reason was that we needed more mobile search records to do log analysis while the other was that experienced users were typical, and had much to say about mobile search behaviors. Participants were identified among the finalists of a search contest hosted by Baidu, China's largest search engine. Electronic questionnaires were sent to their e-mails, and 58 students wrote in reply. We recruited 30 participants among these respondents from all over China.

The participants are all college students (9 male and 21 female), including 21 undergraduates and 9 postgraduates with average age of 21 years old. They come from seven universities with different disciplinary backgrounds such as computer science, information science, library science, linguistics, finance, economics, psychology, and surveying and mapping.

\subsection{Procedure}

By installing AWARE, an Android framework for logging and sharing mobile context information, on participants' smartphones, we were able to collect logs of daily phone use. After signing our participant agreement, participants were asked to promise that they would do mobile search at least once a day. Each of the three laboratory personnel engaged in the experiment managed ten participants. They checked the log records every day to ensure that there was no discontinuity of the AWARE framework and that each participant performed mobile search at least once a day.

Before the experiment, there were short periods of trial operation to test AWARE's function and output. System operation, application usage, keyboard input, and location information were recorded in the experiment. Voice input, accounts and passwords, information received, and browsing history were not recorded. Since AWARE only recorded keyboard input, which participants could control, there was no privacy issue. Because we told the participants what kind of data we collected, they were reassured regarding the privacy of their data.

Journal of Data and Information Science http://www.jdis.org 
Vol. 1 No. 1, 2016

\section{Research Paper}

Structured diaries were distributed to participants by laboratory personnel every day in which they were to identify the most impressive search each day. Information needs, information motivations, search sites, and context were requested in the structured dairy, as well as any interruption of the mobile search session. By collecting the diary records from user phone log files with SQLite Developer, a database administration tool, deeper analysis about users' mobile search behaviors was made possible. Any irregularities or variations in searches were explored in semi-structured interviews with participants.

\subsection{Data Analysis Methods}

In this paper, we define mobile search context as the personal attributes and surroundings of the participants when doing mobile search, including the physical environment (e.g. indoor, outdoor, and specific place) and the social environment (e.g. alone or with others). There are 12 defined dimensions of participants' mobile search context (Table 1), which can be divided into three categories. We get this information from the structured diary and the phone log, and do correlation analysis or difference analysis between context dimensions and mobile search behaviors. The analysis tool we use is Statistical Product and Service Solutions (SPSS 19.0), and analytical methods are cross tabulation and one-way analysis of variance (ANOVA).

Table 1. Dimensions of participants' mobile search context.

\begin{tabular}{lll}
\hline \multicolumn{1}{c}{ Category } & Dimension & \multicolumn{1}{c}{ Definition } \\
\hline $\begin{array}{ll}\text { Personal attributes of the } \\
\text { participant }\end{array}$ & Gender & Sex (male or female) \\
& Age & Participant age \\
& Grade & Freshman, sophomore, junior or senior \\
& Major & Academic field \\
Physical environment of the & Time & Year-month-day-hour-minute-second \\
participant & Place & Indoor or outdoor; specific location \\
& Weather & Summer, rainy or sunny \\
& Device & Phone type and screen size \\
Social environment of the & Activity & Event involved while searching: resting, working, \\
participant & Relations & Accompanied or alone \\
& Importance & Whether the search task is urgent \\
& Portal & Application used \\
\hline
\end{tabular}

\section{Results Analysis}

We explored four aspects of mobile search behaviors: search sessions, search queries, information needs, and emotion changes as follows.

Journal of Data and

A search session is the process in which participants submit one or more queries Information Science to meet a single information need. A search query is the text entered during a search 
session. An information need is a participant's desire to obtain information to satisfy a need. An emotion change refers to a change in a participant's mood upon completion of a mobile search. In this paper, we identify 450 mobile search sessions with definite information needs and emotion changes from the structured diaries.

\subsection{The Impact of Motivation on Different Aspects of Search Behavior}

\subsubsection{Mobile Search Session}

Participants were asked in the structured diaries to identify which motivations defined their most impressive search sessions for each day. We found that it was common for users driven by multiple motivations to finish one mobile search session. We define six motivation categories in our research to meet every search session.

1) Curiosity is a category that reflects the user's interest in an unfamiliar topic that occurs by chance, e.g., while watching a movie and wanting more details on an actor or while chatting with a friend and wanting to prove a point.

2) Time killing is a category that refers to the desire for recreation in one's spare time, where the desired information is useless but recreational, e.g., looking for recreation news or information about famous people.

3) Knowledge is a category that refers to needs derived from the user's profession, e.g. an IT student searching for information about a programming language.

4) Life service includes needs arising in daily life, such as finding directions to a location.

5) Social relations includes two types of searches: seeking contact information for a company with regard to employment information, or false searches, done to appear busy in order to avoid developing a relationship or chatting with somebody in social occasions.

6) Others includes motivations that do not fall into any category above.

Table 2 is a matrix of participants' information motivations. It illustrates searches with one or two motivations. For searches with more than two motivations, we add one for each pair of motivations in that particular search. For example, one participant chose time killing, curiosity, and social relations as the motivations for one mobile search session. We add one to the frequency for each of the following: the relationship between curiosity and social relations, the relationship between time killing and curiosity, and the relationship between time killing and social relations. After all the integers of frequency are added, we calculate the percentages of information motivations by Equation (1) (IntRre is the number of searches driven by a particular motivation):

Journal of Data and Information Science http://www.jdis.org 
Vol. 1 No. 1, 2016

\section{Research Paper}

$$
\text { Percentage }=\frac{\text { IntRre }}{450(\text { mobile search sessions in total })} \text {. }
$$

Table 2. Matrix of search motivations.

\begin{tabular}{lccccccc}
\hline Information & Curiosity & $\begin{array}{c}\text { Time } \\
\text { killing }\end{array}$ & Knowledge & Life service & $\begin{array}{c}\text { Social } \\
\text { relations }\end{array}$ & Others & Total \\
\hline Curiosity & $79(17.56 \%)$ & 38 & 39 & 14 & 3 & 4 & 177 \\
Time killing & $8.44 \%$ & $31(6.89 \%)$ & 22 & 8 & 2 & 5 & 106 \\
Knowledge & $8.67 \%$ & $4.89 \%$ & $88(19.56 \%)$ & 16 & 1 & 7 & 173 \\
Life service & $3.11 \%$ & $1.78 \%$ & $3.56 \%$ & $95(21.11 \%)$ & - & 6 & 137 \\
Social relations & $0.67 \%$ & $0.44 \%$ & $0.22 \%$ & - & $10(2.22 \%)$ & 1 & 17 \\
Others & $0.89 \%$ & $1.11 \%$ & $1.56 \%$ & $1.33 \%$ & $0.22 \%$ & $19(4.22 \%)$ & 40 \\
Total & $39.33 \%$ & $23.56 \%$ & $38.44 \%$ & $30.44 \%$ & $3.78 \%$ & $8.89 \%$ & $650(144.00 \%)$ \\
\hline
\end{tabular}

Note. Integers in Table 2 refer to the frequency of search sessions coming from the motivation in the column. Percentages refer to the ratio of search sessions driven by a certain motivation to total search sessions.

Results show that $71.56 \%$ of all mobile search sessions came from a single search motivation, and $24.44 \%$ came from two search motivations. The remaining $4 \%$ came from three search motivations. Mobile search sessions were mainly motivated by curiosity and knowledge, which accounted for $77.78 \%$ of the total. Search sessions motivated by daily life service and time killing were the second largest group. Even though there was a low rate of searches driven by building or avoiding social relations, we concluded that participants were driven by relatively balanced motivations to perform mobile searches in their daily life, and they relied on mobile search to meet various information needs.

On the other hand, every mobile search session was driven by 1.44 types of motivations on average. Search sessions driven by curiosity, time killing, and knowledge shared high rates of correlation. More specifically, time-killing searches were prone to be influenced by other motivations such as curiosity and knowledge. Search sessions for life service were relatively independent of others.

\subsubsection{Mobile Search Query}

We explore query frequency and repetition pattern to probe the motivation behind mobile search sessions. We add up the queries generated by different types of search motivations. Each search session is presented by a typical query, which means that no matter how many queries appear in a search session, we count only one query here.

Journal of Data and

(i) Search query frequency: Query frequency in different search motivations Information Science is illustrated in Table 3, according to which, participants tended to perform mobile 
searches for satisfying their curiosity, increasing their knowledge, satisfying their daily needs, and killing time. "Others" includes motivations of finishing homework, knowing the date of an examination, etc. This might have something to do with the identity of the participants as college students, who are more eager for new knowledge and have a range of practical needs based on complex academic and social activity schedules that must be met. Searching for information on mobile devices had become an indispensable part of their college life.

Table 3. Motivations and query frequency.

\begin{tabular}{lc}
\hline Motivation & Query frequency (\%) \\
\hline Curiosity & $180(40.00 \%)$ \\
Time killing & $94(20.89 \%)$ \\
Knowledge & $143(31.78 \%)$ \\
Life service & $128(28.44 \%)$ \\
Social relations & $20(4.45 \%)$ \\
Others & $33(7.33 \%)$ \\
\hline
\end{tabular}

Note. Percentage refers to the ratio of queries driven by certain motivation to all queries.

(ii) Repeated search query and motivation: The field of repeated times of queries marked as RT in Table 4 is used to verify different ratios of query reappearances driven by different search motivations, where $9.15 \%$ of all the mobile search queries were repeated from once to 19 times. In total, there were 263 queries repeated in search sessions driven by various motivations and $67.68 \%$ of these queries were repeated only twice. In general, repetition of a query is associated with its type of motivation. Queries coming from search sessions for life service were repeated more times than queries for other motivations. Repeated queries coming from search sessions for curiosity and time killing shared a similar proportion of repeated times, which were less than those of queries for knowledge.

Table 4. Query in different motivations.

\begin{tabular}{lccc}
\hline Motivation & RT $\leq 2(\%)$ & RT $>2(\%)$ & Total $(\%)$ \\
\hline Curiosity & $39(14.83 \%)$ & $9(3.42 \%)$ & $48(18.25 \%)$ \\
Life service & $70(26.62 \%)$ & $40(15.21 \%)$ & $110(41.83 \%)$ \\
Time killing & $26(9.89 \%)$ & $19(7.22 \%)$ & $45(17.11 \%)$ \\
Knowledge & $40(15.21 \%)$ & $17(6.46 \%)$ & $57(21.67 \%)$ \\
Others & $3(1.14 \%)$ & - & $3(1.14 \%)$ \\
Total & $178(67.68 \%)$ & $85(32.32 \%)$ & $263(100 \%)$ \\
\hline
\end{tabular}

Note. RT means repeated times of queries; integer refers to how many queries repeated for certain times; percentage refers to the repetition rate.

Journal of Data and Information Science 


\section{Research Paper}

\subsubsection{Mobile Search Information Need}

The information need types are categorized in reference to Wikipedia ${ }^{\circledR}$, Open Directory Project $(\mathrm{DMOZ})^{\circledR}$, and actual queries formulated by our 30 participants. We created 13 categories to include all queries as noted in Table 5.

Table 5. Categories of information need types.

\begin{tabular}{ll}
\hline \multicolumn{1}{c}{ Category } & \multicolumn{1}{c}{ Examples } \\
\hline Natural science & Chemistry, physics, astronomy \\
Social science & Economics, history, celebrities \\
Culture \& art & Museum, exhibition, performing arts, photography \\
City \& architecture & Chicago, Wuhan, Roman catholic cathedral \\
Education & School, courses, studying abroad \\
Computer skills & Software and hardware, operating skills \\
News \& events & Social news, political news, gossips \\
Medical treatment \& health & Hospitals, diseases, medicare \\
Recreation & Music and videos, restaurants, accommodation \\
Transportation & Roadmap, train and airplane routes \\
Social networking & QQ, WeChat, discuz!, net community \\
Daily shopping & Clothing, books, food, electronics \\
Others & Religions, weather, sports \\
\hline
\end{tabular}

We analyzed the motivation and information need types via cross tabulation and found significant correlations between them, shown in Table 6 . We also analyzed the three significant correlations to understand the information need types under different motivations, as shown in Table 7.

Table 6. Search motivation and information need type.

\begin{tabular}{lc}
\hline \multicolumn{1}{c}{ Motivation } & Correlations $(p$-value $)$ \\
\hline Curiosity & $0.038^{*}$ \\
Time killing & 0.118 \\
Knowledge & $0.020^{*}$ \\
Life service & $0.000^{* *}$ \\
Social relations & 0.358 \\
Social avoidance & 0.910 \\
Others & 0.354 \\
\hline
\end{tabular}

Note. $*<0.05, * * p<0.01$.

Table 7. Top three relationships between search motivation and information need type.

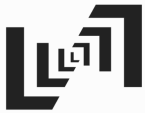

Journal of Data and Information Science

\begin{tabular}{lccc}
\hline Motivation & Top 1 (\%) & Top 2 (\%) & Top 3 (\%) \\
\hline Curiosity & Culture \& art (31.1) & Social science (22.2) & Recreation (6.7) \\
Knowledge & Culture \& art (22.4) & Social science (16.1) & Computer skills (12.6) \\
Life service & Culture \& art (18.0) & Recreation (15.6) & Daily shopping (12.5)
\end{tabular}

Note. Percentage refers to the ratio of certain information need driven by certain type of motivation to all mobile searches.

(2) https://en.wikipedia.org

(3) http://www.dmoz.org 
Tables 6 and 7 indicate that three types of motivations had significant correlations with information needs. Driven by different search motivations, participants appear to have diverse information need types. We concluded that the college students' needs for culture and art information were commonly higher than for other information types. When participants searched to satisfy their curiosity, their information needs for social sciences and recreation were relatively higher. When they searched to increase their knowledge, their information needs for social sciences and computers were also relatively higher. When participants decided to search to satisfy daily life needs, they tended to find information on recreation and shopping.

\subsubsection{Mobile Search Emotion Change}

To understand the different emotion changes under various motivations, we performed a cross tabulation analysis between search motivations and emotion changes, as shown in Table 8.

Table 8. Search motivation and emotion change.

\begin{tabular}{lc}
\hline \multicolumn{1}{c}{ Motivation } & Correlations $(p$-value $)$ \\
\hline Curiosity & 0.444 \\
Time killing & 0.071 \\
Knowledge & 0.075 \\
Life service & 0.121 \\
Social relations & 0.538 \\
Social avoidance & 0.847 \\
Others & $0.000^{* *}$ \\
\hline
\end{tabular}

Note. $*<0.05, * * p<0.01$.

Table 8 shows that search motivation and emotion change did not correlate significantly with each other except for "others" which includes motivations of finishing homework, finding discount information, etc. For example, when users tried to search for discounts or to finish homework, they generally tended to reflect a better mood.

\subsection{The Impact of Context on Different Aspects of Search Behavior}

\subsubsection{Mobile Search Query}

We obtained query frequency data from the structured diaries and the search logs, and counted the query frequency at different periods. Table 9 shows the correlation analysis results, in which we saw that only time and place significantly correlated with query frequency.

Journal of Data and Information Science 


\section{Research Paper}

Table 9. Search query and context.

\begin{tabular}{llc}
\hline \multicolumn{1}{c}{ Category } & Mobile search context dimensions & Correlations $(p$-value $)$ \\
\hline Personal attributes & Gender & 0.351 \\
of the participant & Age & 0.271 \\
& Grade & 0.577 \\
& Major & 0.326 \\
Physical environment & Time & $0.000^{* *}$ \\
around the participant & Place & $0.000^{* *}$ \\
& Weather & 0.699 \\
& Device & 0.154 \\
Social environment & Activity & 0.190 \\
around the participant & Relations & 0.280 \\
& Importance & 0.214 \\
& Portal & 0.881 \\
\hline
\end{tabular}

Note. $*<0.05, * * p<0.01$.

We divided one 24-hour day into four periods - 00:00-06:00, 06:00-12:00, 12:00 $-18: 00$, and 18:00-24:00. We chose this time division to make the length of the four periods equal and make 00:00 the beginning of a day. As mobile search is convenient and instant and the users may do it at any time, we did not take mealtime into consideration. The time division we used might not be optimal as it cut through mealtime, which probably had some influence on the analysis.

Place here contained six specific locations: home or dormitory, workplace, study place, in public, in transit, and on vacation. In order to understand the query frequency in four periods and six locations, we gave two cross tabulations as shown in Tables 10 and 11.

Table 10. Top two relationships between time and query frequency.

\begin{tabular}{cc}
\hline Time & Query frequency (\%) \\
\hline $18: 00-24: 00$ & 44.2 \\
$12: 00-18: 00$ & 40.0 \\
\hline
\end{tabular}

Note. Percentage refers to the ratio of queries users enter during the two periods of time to all queries.

Table 11. Top two relationships between place and query frequency.

\begin{tabular}{lc}
\hline \multicolumn{1}{c}{ Place } & Query frequency (\%) \\
\hline Home or dormitory & 54.7 \\
Study place & 28.7 \\
\hline
\end{tabular}

Note. Percentage refers to the ratio of queries users enter in the two places to all queries.

Journal of Data and Information Science 
Table 10 indicates that about $44.2 \%$ of the queries appeared between 18:00 and 24:00, and $40 \%$ between $12: 00$ and 18:00. Furthermore, the place and query frequency had significant correlations. About $54.7 \%$ of the queries were formulated at home or in the dormitory, and $28.7 \%$ in a study place. This might have something to do with the college students' lifestyle - they often stayed up late and were active in the afternoon, while dormitory and study place were two main places of their college life.

\subsubsection{Mobile Search Information Need}

The information need and the mobile search context are both parts of information search behaviors. The information need is expected to have something to do with the various attributes of the participants and their surroundings. The correlation analysis results are shown in Table 12. If the users tend to search for some information via various portals at the same time, we make analyses of the information need and every portal.

Table 12. Information need and mobile search context.

\begin{tabular}{llc}
\hline \multicolumn{1}{c}{ Category } & Mobile search context dimensions & Correlations $(p$-value $)$ \\
\hline Personal attributes of the & Gender & $0.000^{* *}$ \\
participant & Age & 0.325 \\
& Grade & 0.139 \\
& Major & 0.314 \\
Physical environment of the & Time & 0.232 \\
participant & Place & - \\
& Weather & 0.412 \\
& Device & 0.253 \\
Social environment of the & Activity & $0.002^{* *}$ \\
participant & Relations & 0.577 \\
& Importance & $0.032^{*}$ \\
\end{tabular}

Note. $*<0.05, * * p<0.01$. "A" stands for all correlated, which means all subdivisions of a certain dimension correlated with another dimension or a subdivision of the dimension.

The social environment of the participants had significant correlations with their information needs, while their physical environment did not. To get a better understanding of the correlations, we made the following additional analyses.

(i) Information need types and personal attributes: Table 13 shows that the information need type had significant correlations with gender, which meant that men and women had different information need types. In addition, age, grade, and major did not have any correlations with the information need types.

Journal of Data and Information Science 


\section{Research Paper}

Table 13. Top three relationships between information need and gender.

\begin{tabular}{llll}
\hline Gender & \multicolumn{1}{c}{ Top 1 (\%) } & \multicolumn{1}{c}{ Top 2 (\%) } & \multicolumn{1}{c}{ Top 3 (\%) } \\
\hline Man & Culture \& art (23.0) & Social science (14.1) & Natural science (14.1) \\
Woman & Culture \& art (24.8) & Social science (18.7) & Recreation (10.2) \\
\hline
\end{tabular}

Note. Percentage refers to the ratio of certain gender's information need to all mobile searches.

Table 13 shows that the men and women were mainly concerned about culture and art information. In addition, the women cared more about social sciences and recreation information, whereas the men preferred natural science information. This phenomenon shows that the information need differed between men and women, and that women tended to do mobile search when relaxed.

(ii) Information need type and social environment: The social environment contained current activity, relations, task importance, and portal. Table 12 indicates that all of the dimensions except relations had significant correlations with information need types. To better understand the correlations, we gave cross tabulation in Tables 14-17.

Table 14. Top three relationships between information need and activity.

\begin{tabular}{llll}
\hline Activity & \multicolumn{1}{c}{ Top 1 (\%) } & \multicolumn{1}{c}{ Top 2 (\%) } & \multicolumn{1}{c}{ Top 3 (\%) } \\
\hline On break & Culture \& art (24.4) & Social science (18.1) & Recreation (8.1) \\
Working & Culture \& art (40.0) & Social science (30.0) & Education (15.0) \\
Studying & Culture \& art (23.1) & Natural science (15.7) & Social science (13.9) \\
Shopping & Recreation (57.1) & News \& event (14.3) & Social science (14.3) \\
Traveling & Recreation (28.6) & Culture \& art (21.4) & Social science (14.3) \\
Others & Culture \& art (22.6) & Social science (16.1) & Natural science (12.9) \\
\hline
\end{tabular}

The activity and information need types significantly correlated with each other (Table 14). Participants cared about recreation information when taking a break, education information when working, and natural science information when studying. In addition, participants preferred culture and art information when traveling.

The search task can be important or urgent sometimes. From Table 12, we knew that search task importance significantly correlated with the information need types. When searching for social science-especially economics and celebritiesparticipants considered it to be important, while culture and art were considered unimportant.

Table 15. Top three relationships between information need and importance.

\begin{tabular}{llll}
\hline \multicolumn{1}{c}{ Task importance } & \multicolumn{1}{c}{ Top 1 (\%) } & \multicolumn{1}{c}{ Top 2 (\%) } & \multicolumn{1}{c}{ Top 3 (\%) } \\
\hline Important & Social science (24.7) & Culture \& art (16.7) & Recreation (11.4) \\
Neutral & Culture \& art (27.5) & Social science (10.8) & Natural science (10.3) \\
Unimportant & Social science (28.0) & Culture \& art (25.8) & City \& architecture (8.0) \\
\hline
\end{tabular}


Search portal and information need types were correlated, but the prominent correlation between each search portal and information need types was slightly different, as shown in Tables 16 and 17.

Table 16. Information need and search portal.

\begin{tabular}{lc}
\hline \multicolumn{1}{c}{ Search portal } & Correlations $(p$-value $)$ \\
\hline Browser & $0.000^{* *}$ \\
Video software & $0.007^{* *}$ \\
Music software & $0.000^{* *}$ \\
Transportation software & $0.000^{* *}$ \\
Social software & $0.000^{* *}$ \\
Shopping software & $0.000^{* *}$ \\
Application store & $0.000^{* *}$ \\
Education software & $0.023^{*}$ \\
\hline
\end{tabular}

Note. $*<0.05, * * p<0.01$.

Table 17. Top three relationships between search portal and information need types.

\begin{tabular}{llll}
\hline \multicolumn{1}{c}{ Search portal } & \multicolumn{1}{c}{ Top 1 (\%) } & \multicolumn{1}{c}{ Top 2 (\%) } & \multicolumn{1}{c}{ Top 3 (\%) } \\
\hline Browser & Culture \& art (23.6) & Social science (18.4) & Natural science (9.2) \\
Video software & Culture \& art (63.2) & Recreation (10.5) & Social science (5.3) \\
Music software & Culture \& art (100) & - & - \\
Transportation software & Transportation (47.6) & Recreation (33.3) & Others (14.3) \\
Social software & Social science (45.9) & Social networking (16.2) & Culture \& art (13.5) \\
Shopping software & Daily shopping (57.1) & Recreation (35.7) & Social networking (3.6) \\
Application store & Computer skills (80.0) & Recreation (20.0) & - \\
Education software & Culture \& art (66.7) & Natural science (25.0) & Others (8.3) \\
\hline
\end{tabular}

As the above tables show, mobile search portal and the information need had significant correlations. Besides culture, art, and social science, the information searched by browsers was also about natural science, so browsers could serve as a good search portal for participants' daily studying. The information searched by video application and music application was mostly about culture, art, and recreation, and this conformed to the resources characteristics of the search portal itself. When setting traveling and traffic, social networking, shopping, and learning software as portals, the information need types were consistent with the characteristics of those search portals. When participants were in the application stores, they generally searched computers and recreation applications.

\subsubsection{Mobile Search Emotion Change}

Emotion change in mobile search meant the change of user emotion after searching for information on a mobile device. It is divided into three categories: better, worse, and no change.

From Table 18, we knew that among the 12 dimensions, only gender, task importance, and portal significantly correlated with emotion change. We also saw

Journal of Data and Information Science 


\section{Research Paper}

that the social environment more closely correlated with emotion change than the other two context categories. We examined every search portal with respect to emotion change and learned that all the search portals correlated with emotion change in mobile search to some degree.

Table 18. Emotion change and context.

\begin{tabular}{llc}
\hline \multicolumn{1}{c}{ Category } & Mobile search context dimensions & Correlations $(p$-value $)$ \\
\hline Personal attributes & Gender & $0.013^{*}$ \\
of the participant & Age & 0.342 \\
& Grade & 0.451 \\
& Major & 0.182 \\
Physical environment & Time & 0.147 \\
of the participant & Place & - \\
& Weather & 0.723 \\
& Device & 0.247 \\
Social environment & Activity & 0.108 \\
of the participant & Relations & 0.230 \\
& Importance & $0.000^{* *}$ \\
\hline
\end{tabular}

Note. "P" stands for partially correlated, i.e., a subdivision of a certain dimension correlates with another dimension or a subdivision of the dimension.

(i) Emotion change and personal attributes: Among the four dimensions of personal attributes, only gender had significant correlations with emotion change; we made further analysis of the relationships between them.

Table 19 shows that the men's emotion change was more significant than that of the women. This might have something to do with the information for which they searched. For example, the women tended to search for recreation information, while the men preferred natural science. Men became happier when they got knowledge, while the women focused more on fun.

Table 19. Relationships between gender and emotion change.

\begin{tabular}{lccc}
\hline Gender & Better (\%) & Worse (\%) & Neither (\%) \\
\hline Man & 42.2 & 10.4 & 47.4 \\
Woman & 28.9 & 9.2 & 61.9 \\
\hline
\end{tabular}

(ii) Emotion change and social environment: As Table 20 shows, emotion change of the participants and the importance of the tasks significantly correlated with each other. Emotion change appeared more obvious as the search task became

Journal of Data and more important or urgent. We concluded that the emotion change varies depending Information Science on whether the important information need is satisfied. 
How Users Search the Mobile Web: A Model for Understanding the Impact of Motivation and Context on Search Behaviors

Table 20. Relationships between emotion change and task importance.

\begin{tabular}{lccc}
\hline \multicolumn{1}{c}{ Task importance } & Better (\%) & Worse (\%) & Neither (\%) \\
\hline Important & 45.6 & 17.5 & 36.8 \\
Neutral & 33.8 & 7.8 & 58.3 \\
Unimportant & 20.5 & 5.3 & 74.2 \\
\hline
\end{tabular}

We analyzed the correlation between every search portal and emotion change. It turned out that some of the search portals significantly correlated with emotion change. As seen in Tables 21 and 22, 89\% of the participants' mood did not become worse when searching through a browser. This illustrates the utility of the browser for the participants. When using social software, the participants did not have dramatic changes in emotion.

Table 21. Emotion change and search portal.

\begin{tabular}{lc}
\hline \multicolumn{1}{c}{ Search portal } & Correlations $(p$-value $)$ \\
\hline Browser & $0.032^{*}$ \\
Video software & 0.198 \\
Music software & 0.321 \\
Transportation software & 0.740 \\
Social software & $0.021^{*}$ \\
Shopping software & 0.586 \\
Application store & 0.390 \\
Education software & 0.052 \\
\hline
\end{tabular}

Note. $*<0.05, * * p<0.01$.

Table 22. Relationships between emotion change and search portal.

\begin{tabular}{lccc}
\hline Search portal & Better (\%) & Worse (\%) & Neither (\%) \\
\hline Browser & 35.0 & 11.0 & 54.0 \\
Social software & 13.5 & 8.1 & 78.4 \\
\hline
\end{tabular}

\subsection{Context Dimensions}

The dimensional context of mobile search may have correlations between any two dimensions. We explored every significant correlation to better understand how the mobile search context affected mobile search behaviors. The analysis results were presented in Tables $23-34$.

\subsubsection{Personal Attributes and Physical Environment}

The personal attributes of the participants and the physical environment did not correlate with each other except for gender and place. We made further analysis of the two dimensions (Tables 24 and 25).

Journal of Data and Information Science 


\section{Research Paper}

Table 23. Personal attributes and physical environment.

\begin{tabular}{|c|c|c|c|c|c|c|c|c|c|}
\hline \multirow{2}{*}{ Context } & & \multicolumn{4}{|c|}{ Personal attributes ( $p$-value) } & \multicolumn{4}{|c|}{ Physical environment ( $p$-value) } \\
\hline & & Gender & Age & Grade & Major & Time & Place & Weather & Device \\
\hline \multirow{4}{*}{$\begin{array}{l}\text { Personal } \\
\text { attributes }\end{array}$} & Gender & - & - & - & - & 0.673 & $\mathrm{P}$ & - & - \\
\hline & Age & - & - & - & - & 0.347 & 0.748 & - & - \\
\hline & Grade & - & - & - & - & 0.284 & 0.345 & - & - \\
\hline & Major & - & - & - & - & 0.529 & 0.653 & - & - \\
\hline \multirow{4}{*}{$\begin{array}{l}\text { Physcial } \\
\text { environment }\end{array}$} & Time & 0.673 & 0.347 & 0.284 & 0.529 & - & 0.354 & 0.736 & - \\
\hline & Place & $\mathrm{P}$ & 0.748 & 0.345 & 0.653 & 0.354 & - & 0.415 & 0.866 \\
\hline & Weather & - & - & - & - & 0.736 & 0.415 & - & 0.593 \\
\hline & Device & - & - & - & - & - & 0.866 & 0.593 & - \\
\hline
\end{tabular}

Note. "P" stands for partially correlated, i.e., a subdivision of a certain dimension correlates with another dimension or a subdivision of the dimension.

Table 24. Gender and search place.

\begin{tabular}{ccccccc}
\hline & $\begin{array}{c}\text { At home or dormitory } \\
(p \text {-value })\end{array}$ & $\begin{array}{c}\text { In workplace } \\
(p \text {-value })\end{array}$ & $\begin{array}{c}\text { In study place } \\
(p \text {-value })\end{array}$ & $\begin{array}{c}\text { In public } \\
(p \text {-value })\end{array}$ & $\begin{array}{c}\text { On the way } \\
(p \text {-value }\end{array}$ & $\begin{array}{c}\text { On vacation } \\
(p \text {-value })\end{array}$ \\
\hline Gender & 0.967 & 0.912 & $0.000^{* *}$ & $0.006^{* *}$ & $0.008^{* *}$ & $0.017^{*}$ \\
\hline
\end{tabular}

Note. $*<0.05, * * p<0.01$.

Table 25. Relationships between gender and search place.

\begin{tabular}{lcccc}
\hline & In study place (\%) & In public (\%) & On the way (\%) & On vacation (\%) \\
\hline Man & 43.7 & 3.0 & 4.4 & 0 \\
Woman & 22.2 & 10.8 & 12.7 & 4.1 \\
\hline
\end{tabular}

Tables 24 and 25 show that the four kinds of places were closely connected with gender. Specifically, men tended to do mobile search in study areas, whereas women easily performed mobile search when they were on the way to places, in public, and on vacation. Mobile search was more popular in women's daily life, while men preferred to use mobile devices when studying.

\subsubsection{Personal Attributes and Social Environment}

The detailed correlation results are listed in Table 26.

(i) Gender and activity: Among the personal attributes of the participants, gender and current activity had significant correlations with each other, which meant that men were more likely to do mobile search when studying, whereas women often carried out mobile search when they were on a break. This shows some of the different functions of mobile search based on gender (Table 27).

Journal of Data and Information Science 
How Users Search the Mobile Web: A Model for Understanding the Impact of Motivation and Context on Search Behaviors

Dan Wu et al.

Table 26. Personal attributes and social environment.

\begin{tabular}{|c|c|c|c|c|c|c|c|c|c|}
\hline \multirow{2}{*}{\multicolumn{2}{|c|}{ Context }} & \multicolumn{4}{|c|}{ Personal attributes ( $p$-value) } & \multicolumn{4}{|c|}{ Social environment ( $p$-value) } \\
\hline & & Gender & Age & Grade & Major & Activity & Relations & Importance & Portal \\
\hline \multirow{4}{*}{$\begin{array}{l}\text { Personal } \\
\text { attributes }\end{array}$} & Gender & - & - & - & - & $0.000^{* *}$ & 0.138 & 0.116 & $\mathrm{P}$ \\
\hline & Age & - & - & - & - & 0.357 & 0.231 & 0.573 & 0.717 \\
\hline & Grade & - & - & - & - & 0.399 & 0.786 & 0.396 & 0.202 \\
\hline & Major & - & - & - & - & 0.610 & 0.577 & 0.150 & 0.598 \\
\hline \multirow{4}{*}{$\begin{array}{l}\text { Social } \\
\text { environment }\end{array}$} & Activity & $0.000^{* *}$ & 0.357 & 0.399 & 0.610 & - & $0.000^{* *}$ & $0.000^{* *}$ & - \\
\hline & Relations & 0.138 & 0.231 & 0.786 & 0.577 & $0.000^{* *}$ & - & 0.633 & 0.171 \\
\hline & Importance & 0.116 & 0.573 & 0.396 & 0.150 & $0.000^{* *}$ & 0.633 & - & 0.573 \\
\hline & Portal & $\mathrm{P}$ & 0.717 & 0.202 & 0.598 & - & 0.171 & 0.573 & - \\
\hline
\end{tabular}

Note. * $<0.05, * * p<0.01$. "P" stands for partially correlated, i.e., a subdivision of a certain dimension correlates with another dimension or a subdivision of the dimension.

Table 27. Relationships between gender and activity.

\begin{tabular}{llccc}
\hline & & \multicolumn{3}{c}{ Activity } \\
\cline { 3 - 5 } & & Top 1 (\%) & Top 2 (\%) & Top 3 (\%) \\
\hline \multirow{2}{*}{ Gender } & Man & On break (55.6) & Studying (37.0) & Others (4.4) \\
& Woman & On break (61.9) & Studying (18.4) & Others (7.9) \\
\hline
\end{tabular}

(ii) Gender and search portal: We found that gender and portal were partly correlated (Table 28). Transportation software, social software, and shopping software significantly correlated with gender. The women were more likely to do mobile search via social software and shopping software. This phenomenon might have something to do with the different information concerns of men and women, bringing them to choose corresponding search portals.

Table 28. Gender and search portal.

\begin{tabular}{lc}
\hline \multicolumn{1}{c}{ Search portal } & Gender $(p$-value $)$ \\
\hline Browser & $0.001^{* *}$ \\
Video software & 0.239 \\
Music software & 0.627 \\
Transportation software & $0.036^{*}$ \\
Social software & $0.000^{* *}$ \\
Shopping software & $0.021^{*}$ \\
Application store & 0.624 \\
Education software & 0.798 \\
\hline
\end{tabular}

Note. $*<0.05, * * p<0.01$.

(iii) Activity and relations: Current activity and relations significantly correlated with each other, as shown in Table 29. The participants were often alone when they were on a break, studying, or working while shopping, traveling, and

Journal of Data and Information Science 


\section{Research Paper}

doing other things when with friends. This might have something to do with the characteristics of the current activity.

Table 29. Relationships between activity and relations.

\begin{tabular}{lcc}
\hline & Alone $(\%)$ & With others $(\%)$ \\
\hline On break & 49.3 & - \\
Working & 55.0 & - \\
Studying & 50.0 & - \\
Shopping & - & 42.9 \\
Traveling & - & 85.7 \\
Others & - & 38.7 \\
\hline
\end{tabular}

(iv) Activity and importance: Current activity and importance of search task significantly correlated with each other, as shown in Table 30. The search tasks performed were usually unimportant or non-urgent when participants were on a break and doing casual things, while the tasks were important when they were working, studying, and traveling.

Table 30. Relationships between activity and task importance.

\begin{tabular}{lccc}
\hline & Important (\%) & Unimportant (\%) & Neutral (\%) \\
\hline On break & 15.9 & 38.1 & 45.9 \\
Working & 45.0 & 10.0 & 45.0 \\
Studying & 40.7 & 11.1 & 48.1 \\
Shopping & 28.6 & 0 & 71.4 \\
Traveling & 50.0 & 21.4 & 28.6 \\
Others & 29.0 & 38.7 & 32.3 \\
\hline
\end{tabular}

\subsubsection{Physical Environment and Social Environment}

The detailed correlation results are listed in Table 31.

Table 31. Physical environment and social environment.

\begin{tabular}{|c|c|c|c|c|c|c|c|c|c|}
\hline \multirow{2}{*}{\multicolumn{2}{|c|}{ Context }} & \multicolumn{4}{|c|}{ Physical environment ( $p$-value) } & \multicolumn{4}{|c|}{ Social environment ( $p$-value) } \\
\hline & & Time & Place & Weather & Device & Activity & Relations & Importance & Portal \\
\hline \multirow{4}{*}{$\begin{array}{l}\text { Physical } \\
\text { environment }\end{array}$} & Time & - & - & 0.736 & - & 0.593 & 0.586 & 0.206 & - \\
\hline & Place & - & - & 0.415 & 0.866 & $0.000^{* *}$ & A & - & - \\
\hline & Weather & 0.736 & 0.415 & - & 0.593 & 0.559 & 0.374 & 0.412 & - \\
\hline & Device & - & 0.866 & 0.593 & - & 0.137 & 0.037 & 0.061 & 0.186 \\
\hline \multirow{4}{*}{$\begin{array}{l}\text { Social } \\
\text { environment }\end{array}$} & Activity & 0.593 & $0.000^{* *}$ & 0.559 & 0.137 & - & $0.000^{* *}$ & $0.000^{* *}$ & - \\
\hline & Relations & 0.586 & A & 0.374 & 0.347 & $0.000^{* * *}$ & - & 0.633 & 0.171 \\
\hline & Importance & 0.206 & - & 0.412 & 0.631 & $0.000^{* * *}$ & 0.633 & - & 0.573 \\
\hline & Portal & - & - & - & 0.186 & - & 0.171 & 0.573 & - \\
\hline
\end{tabular}

Note. $*<0.05, * * p<0.01$. "A" stands for all correlated, which means all subdivisions of a certain dimension correlate with another dimension or a subdivision of the dimension.

Journal of Data and 
How Users Search the Mobile Web: A Model for Understanding the Impact of Motivation and Context on Search Behaviors

(i) Place and activity: As shown in Table 31, the search places and current activity all correlated with each other. Further analysis is shown in Table 32. The activities the participants were engaged in depended greatly on the function of the place. For example, they were often taking a break when at a friend's home or dormitory, while studying when in a study place.

Table 32. Relationships between place and activity.

\begin{tabular}{lllcl}
\hline & \multicolumn{3}{c}{ Activity } \\
\cline { 3 - 4 } & & \multicolumn{1}{c}{ Top 1 (\%) } & Top 2 (\%) & Top 3 (\%) \\
\hline Place & At home or dormitory & On break (80.5) & Studying (13.4) & Working (3.7) \\
& In workplace & Working (50.0) & On break (31.3) & Studying (12.5) \\
& In study place & Studying (58.9) & On break (34.1) & Others (4.7) \\
& In public & On break (44.7) & Others (28.9) & Shopping (10.5) \\
& On the way & On break (50.0) & Traveling (23.9) & Others (23.9) \\
On vacation & Traveling (84.6) & On break (15.4) & - \\
\hline
\end{tabular}

The search place and the relations of the participant were significantly correlated. To make it as specific as possible, we performed across tabulation analysis between every place and the relations (Tables 33 and 34).

Table 33. Place and relations.

\begin{tabular}{lc}
\hline \multicolumn{1}{c}{ Place } & Relations $(p$-value $)$ \\
\hline At home or dormitory & $0.001^{* *}$ \\
In workplace & $0.000^{* *}$ \\
In study place & $0.000^{* *}$ \\
In public & $0.037^{*}$ \\
On the way & $0.001^{* *}$ \\
On vacation & $0.000^{* *}$ \\
\hline
\end{tabular}

Note. $*<0.05, * * p<0.01$.

Table 34. Relationships between place and relations.

\begin{tabular}{llll}
\hline & \multicolumn{1}{c}{ Top 1(\%) } & \multicolumn{1}{c}{ Top 2 (\%) } & \multicolumn{1}{c}{ Top 3 (\%) } \\
\hline At home or dormitory & Alone (50.4) & With classmates (23.2) & With friends (19.5) \\
In workplace & Alone (43.8) & With friends (31.3) & With workmates (12.5) \\
In study place & Alone (47.3) & With classmates (38.0) & With friends (10.9) \\
In public & With friends (42.1) & Alone (26.3) & With classmates (23.7) \\
On the way & Alone (60.9) & With friends (32.6) & With strangers (7.7) \\
On vacation & With friends (92.3) & Alone (7.7) & -
\end{tabular}

As shown in Table 33, there are significant correlations between search places and current relations when doing mobile search and Table 34 shows the correlations more specifically. The participants were alone when at home or in a dormitory, in 
Vol. 1 No. 1, 2016

\section{Research Paper}

their workplace, and in a study place. They were often with their friends when in public or on vacation. This has something to do with the characteristics and functions of the places.

\subsection{Mobile Search Behavior Model Based on Motivations and Context}

Based on the analysis of the impact of mobile search motivations and context on search behaviors, we built a multi-dimensional model of mobile search behaviors and the factors that influence it (Figure 1).

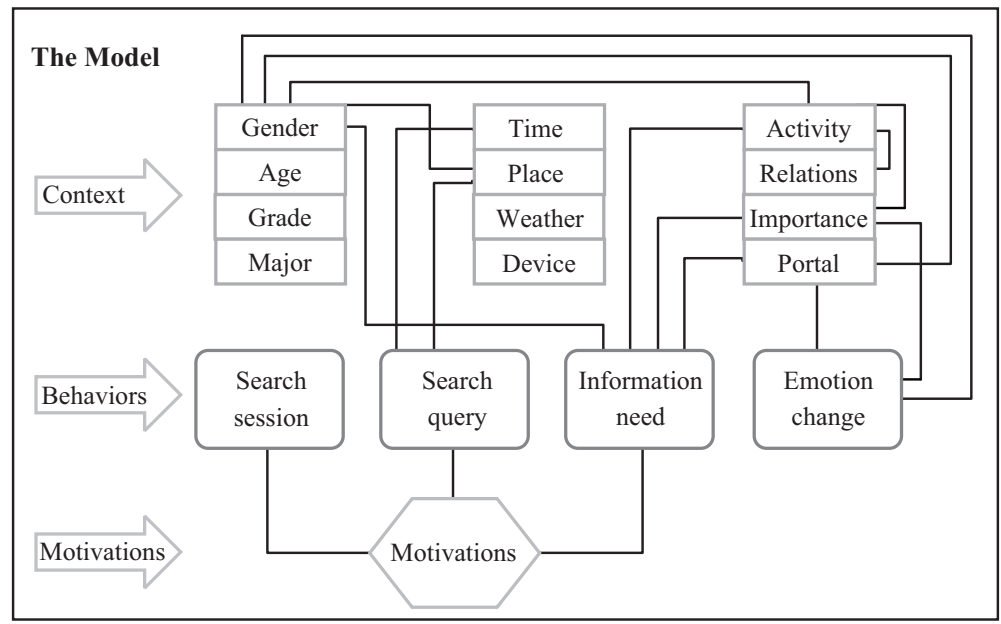

Figure 1. The motivation- and context-based mobile search behavior model.

Search motivations affect mobile search behaviors in terms of mobile search sessions, mobile search queries, and information needs. In contextual dimensions, the time and place of mobile search correlate with the mobile search query. In addition, gender, search activity, search task importance, and portal all have significant correlations with the information need types. The correlations among the contextual dimensions are also significant. Gender, time, place, search activity, relations, task importance, and portal correlate with each other in several ways, and the other dimensions have no significant correlations. In short, mobile search is a successive process involving mobile search motivation, context and mobile search behaviors.

\section{Discussion}

\subsection{Mobile Search Behaviors and Information Motivations}

Journal of Data and Information Science

From the listed findings in the mobile search session and information motivation, we note it is hard to differentiate curiosity-based search sessions from those based 
on recreation or learning, because users tended to find positive reasons for time killing and recreation. Another possibility is that it is difficult to distinguish among the three motivations in participants' cognition because recreation and learning are at times incorporated or have blurred lines between activities and are now harder to recognize or categorize than before. Participants may need information to satisfy curiosity, to increase knowledge, or to enjoy information and recreation, and users rely on mobile phones to meet all these needs. However, life service emphasizes the instant usefulness of information, such as travel information and data to help users make comparisons and decisions on consumer and other activities.

When it comes to mobile search query, we find that queries coming from life service motivations are generally uniform and hard to change, indicating that information needed for life service is more conventional than mobile searches driven by curiosity, time killing, and learning.

The motivation for doing mobile search has significant correlations with the information need types, especially when driven by curiosity, learning, and enjoying life service. However, the correlation between emotion change and motivation is not strong in this study. As can be expected, under different search motivations, users appeared to need diverse information types. The need for culture and art is shown to be the highest. College students are commonly committed to their studies, however, and information searches are often motivated by curiosity and learning.

\subsection{Mobile Search Behaviors and Search Context}

The mobile search context has significant correlations with mobile search behaviors, which can be explained from four aspects: the search session, the search query, the information need, and the emotion change after searching. There may be some reasons for these behaviors hidden behind the correlations. As use of the mobile Internet is becoming pervasive from early ages, college students have gained a good command of it, and thus use mobile search every day. Also, college students are familiar with the mobile Internet, and their daily life on campus reflects more diversity in terms of interests, lifestyles, and opportunities, and their schedules while often full will have flexibility. As college students also place high importance on social activities and allow for discretionary time in their schedules, they have strong and diverse information needs. In addition, we notice that browsers are found to be a better choice than other portals for mobile search. Search engines accessed through browsers are very general, and useful for all kinds of searches.

There are also significant correlations among the different mobile search context dimensions, such as gender with place, search activity, and portal. Men and women

Journal of Data and Information Science 


\section{Research Paper}

exhibited different daily routine priorities and information concerns, as women tended to stay at home more than men who often preferred to go outside. What's more, women in this study were more engaged in social and consumer activities, while men's interests tended towards the technical and scientific concerns. Furthermore, college students tended to do mobile search in certain situations, and often their searches are triggered by certain activities. For instance, they tended to search for information for learning when studying something new.

\section{Conclusion}

Our study adopts a free experiment to learn about college students' mobile search behaviors in their daily life, where participants contributed information on their phone logs and diaries to help us understand how search motivations and context influence their mobile search behaviors. Conclusions follow.

About three-fourths of the mobile search sessions are motivated by a single motivation, while a quarter are motivated by multiple motivations. Different search motivations have the tendency to cross and converge, especially among searches driven by curiosity, time killing, and learning. Queries in search sessions influenced by the former three motivations tend to be repeated less frequently than queries in life service, which suggests that the former three motivations stimulate more types of information needs, while the life service motivation is more stable and uniform. Information needs are found to be mainly driven by three types of motivation: curiosity, learning, and life service. No significant correlations are found between emotion changes and motivations.

We find that information search behaviors significantly correlate with the multidimensional context and the motivation. Gender, search activity, task importance, and portal all have significant correlations with the information need type, and the information needs driven by different motivations are diverse. Furthermore, the correlations among the dimensional context are significant. Gender, time, place, search activity, relations, search task importance, and portal dimensions are all correlated in several ways, and the other dimensions have no significant correlations.

A limited number of participants and brief experimental duration are the main limitations in our research. Given a shoestring budget and the need for remuneration of every participant, the experiment lasted only half a month. In future studies, we will make comparison with studies of desktop search behaviors, and focus on the impact of subjective factors on mobile search behaviors. We may also look at sample groups other than college students and in other locations to make the study more applicable across various populations. 
How Users Search the Mobile Web: A Model for Understanding the Impact of Motivation and Context on Search Behaviors

\section{Acknowledgements}

This work is jointly supported by the Wuhan International Science and Technology Cooperation Fund (Grant No.: 2015030809020371) and the Wuhan University Youth Fund of Humanities and Social Sciences.

\section{Author Contributions}

D.Wu (woodan@whu.edu.cn, corresponding author) was responsible for study design, manuscript review, and the integrity of the study, and made a final approval of the manuscript; M. Zhu (zhuman0521@163.com) was responsible for experimental studies and data analysis and interpretation, wrote the first draft of the manuscript and revised the manuscript. A.H. Ran (ranaihuasim@whu.edu.cn) was responsible for experimental studies and data analysis and interpretation, and revised the manuscript.

\section{References}

Bilandzic, M., \& Foth, M. (2013). Libraries as coworking spaces understanding user motivations and perceived barriers to social learning. Library Hi Tech, 31(2): 254-273.

Church, K., \& Oliver, N. (2011).Understanding mobile web and mobile search use in today's dynamic mobile landscape. In Proceedings of Mobile HCI 2011 Stockholm, 13th International Conference on Human Computer Interaction with Mobile Devices \& Services (pp. 67-76). Stockholm, Sweden: Swedish Institute of Computer Science.

Ciampa, K. (2014). Learning in a mobile age: An investigation of student motivation. Journal of Computer Assisted Learning, 30(1): 82-96.

Chang, J., \& Yang, J.M. (2009). An empirical study on the relationship among baidupedia users' participating behavior and motivations (in Chinese). Studies in Science of Science, 27(8): $1213-1219$.

Gasimov, A., Magagna, F., \& Sutanto, J. (2010). CAMB: Context-aware mobile browser. Proceedings of the 9th International Conference on Mobile and Ubiquitous Multimedia (pp. 22:122:5). Limassol, Cyprus: Cyprus University of Technology and Frederick University.

Huizenga, J., Admiraal, W., Akkerman, S., \& ten Dam, G. (2009). Mobile game-based learning in secondary education: Engagement, motivation, and learning in a mobile city game. Journal of Computer Assisted Learning, 25(4): 332-344.

Kamvar, M., \&Baluja, S. (2006). A large scale study of wireless search behavior: Google mobile search. In Proceedings of CHI 2006 Montréal, Special Interest Group on Computer-Human Interaction Conference (pp. 701-709). Montréal, Canada: Convention Center.

Kim, Y., \& Adler, M. (2015). Social scientists' data sharing behaviors: Investigating the roles of individual motivations, institutional pressures, and data repositories. International Journal of Information Management, 35(4): 408-418.

Kiseleva, J. (2015). Using contextual information to understand searching and browsing behavior. In Proceedings of SIGIR'15 Santiago, 38th Special Interest Group on Information Retrieval Conference (pp. 1059). Santiago, Chile: Association for Computing Machinery.

Journal of Data and Information Science 


\section{Research Paper}

Kong, W. Z., Li, R., Jie, L., Zhang, A., Chang, Y., \& Allan J. (2015). Predicting search intent based on pre-search context. In Proceedings of SIGIR'15 Santiago, 38th Special Interest Group on Information Retrieval Conference (pp. 503-512). Santiago, Chile: Association for Computing Machinery.

Liu, X., \& Wu, W. (2015). Learning context-aware latent representations for context-aware collaborative filtering. In Proceedings of SIGIR'15 Santiago, 38th Special Interest Group on Information Retrieval Conference (pp. 887-890). Santiago, Chile: Association for Computing Machinery.

Malhotra, Y., Galletta, D.F., \& Kirsch, L.J. (2008). How endogenous motivations influence user intentions: beyond the dichotomy of extrinsic and intrinsic user motivations. Journal of Management Information Systems, 25(1): 267-300.

Park, E., \& Ohm, J. (2014). Factors influencing users' employment of mobile map services. Telematics and Informatics, 31(2): 253.

\section{(2) $(1) \Theta$}

This license allows readers to redistribute, commercially and non-commercially, as long as it is passed along unchanged and in whole, with credit to the original Author(s) and the Contribution. Please read the full license for further details at http://creativecommons.org/licenses/by/4.0/

Journal of Data and Information Science 